\title{
Condition Monitoring of Centrifugal Pumps Based on Pressure Measurements
}

\author{
Csanád Kalmár ${ }^{1 *}$, Ferenc Hegedűs \\ 1 Department of Hydrodynamic Systems, Faculty of Mechanical Engineering, Budapest University of Technology and Economics, \\ H-1111 Budapest, Múegyetem rkp. 3., Hungary \\ * Corresponding author, e-mail: cskalmar@hds.bme.hu
}

Received: 01 March 2018, Accepted: 01 February 2019, Published online: 18 March 2019

\begin{abstract}
The purpose of the present study is the investigation of condition of centrifugal pumps via pressure signals. Instead of vibration measurement on the housings that is widely used in industry, our method is based on pressure signal measurement on the pressure side of the pump. Fourier transforming such a signal can get us to make conclusions about the behavior of the pump. By changing the operating point along a characteristic curve, we can create waterfall diagrams that provide useful information about the pump at constant rotational speed. For example, it is possible to differentiate the mechanical and the hydrodynamical effects predicting the occurrence of many constructional failures (such as unbalance, angular misalignment, bearing misalignment, motor instability, etc.); thus, preventing heavy damage of the equipment.
\end{abstract}

\section{Keywords}

condition monitoring, centrifugal pump, spectral analysis, diagnostics, waterfall diagram

\section{Introduction}

Centrifugal pumps are one of the mostly used turbomachines in the industry and in households as well [1]. Because of the massive application and the increasing aim for reducing energy consumption, it is necessary to develop continuous condition monitoring techniques on pumps. By detecting several productions or mounting failures, we have the chance to intervene in time; thus, granting good operation efficiency or preventing serious accidents.

In the industry, the widely used method for vibration monitoring is the measurement of acceleration on the bearings $[2,3]$. This technique is mostly applied because of its simple and straightforward utilization, but has a quite big disadvantage that some surface treatment is necessary to mount the sensors. Such treatment causes the loss of the guarantee, which is unacceptable in most cases. This problem can be avoided by applying a method based on pressure signal measurement on the pressure side of a pump, which is easy to implement in most cases.

Pressure signal measurements are mainly used in order to detect cavitation in pipeline systems [4]. Ĉdina [5] made measurements based on acoustic signals, and found correlation between acoustic emission and cavitation. However, according to the best knowledge of the authors, condition monitoring techniques based on pressure measurements has not yet been published in the literature. Therefore, the main aim of the present study is to develop and test such a method by comparing the results obtained on two different centrifugal pumps having very different health conditions.

\section{The test rigs}

For simplicity, the two pumps are indicated with signs " $A$ " and " $B$ ". Pump " $A$ " (BMS 24/48) is a much used, quite old machine. Its driving motor and the pump itself are assembled manually; consequently, parallel or angular misalignments and other mechanical failures can easily occur. In contrast, pump " $B$ " (Grundfos TPE 65-340/2) is a much newer, more compact equipment with much higher precision requirements. The number of blades is 7 and 6 for pump " $A$ " and " $B$ ", respectively.

Fig. 1 shows the sketch of the two test rigs. The pumps $(P)$ circulate water from the reservoirs $(R)$. The rotational speed of pump " $B$ " can be set with a frequency converter. However, in the case of pump " $A$ ", the rotational speed of the driving motor is regulated via a thyristor. Consequently, the control system can become unstable at certain regions of rotational speeds. This can be identified in the spectra 

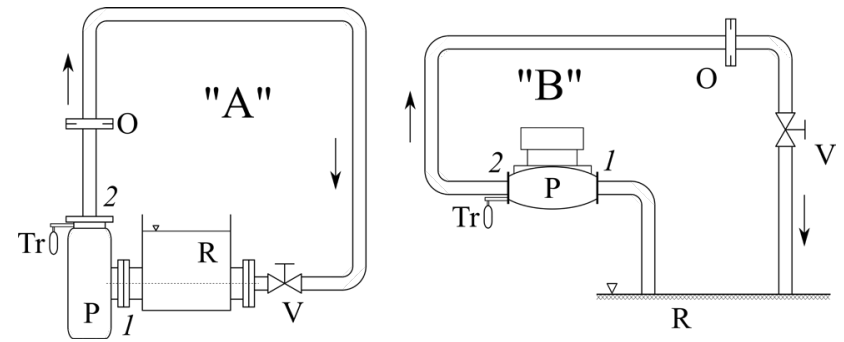

Fig. 1 The sketch of the two measurement test rigs, with pump " $A$ " on the left and pump " $B$ " on the right. The pumps $(P)$ circulate water from the tanks $(R)$ through a throttle valve $(V)$. The pressure transducers $(T r)$ are placed at the pressure side of the pumps.

of the measured pressure signal as a frequency modulation discussed in more details later. The flow rate can be adjusted with a throttle valve $(V)$ and measured by a standard orifice plate $(O)$. The same pressure transducer $(T r)$ is mounted on the pressure side of each pump. The calibration diagram of the pressure transducer (HBM P6A) is shown in the Appendix (see Fig. A1), together with its confidence bands corresponding to $95 \%$ confidence level. The signal of the transducer is sampled with a data acquisition system (HBM Spider 8), the sampling rate of the transducer was $9600 \mathrm{~Hz}$.

The quantities for the characteristic curves are calculated as follows. It is known that the flow rate of the system is proportional to the square root of the pressure drop on a standard measuring orifice [6]. This orifice is an equipment with standard diameter, and prescribed straight segments behind and ahead, too. The flow rate $(Q)$ through the orifice is calculated as

$Q=\alpha \frac{d^{2} \pi}{4} \sqrt{\frac{2 \cdot \Delta p_{o}}{\rho}}$,

where $\alpha$ is the discharge coefficient calculated by an iterative method according to the standard. $d=0.03 \mathrm{~m}$ is the diameter of the orifice for both pumps, $\rho=998 \mathrm{~kg} / \mathrm{m}^{3}$ is the density of water at ambient temperature of $T_{0}=22{ }^{\circ} \mathrm{C}$. The pressure drop $\Delta p_{0}$ on the orifice is measured by a single-tube mercury filled manometer.

The head of the pumps $(H)$ are calculated as

$H=\frac{p_{2}-p_{1}}{\rho g}+\frac{c_{2}^{2}-c_{1}^{2}}{2 g}+h_{2}-h_{1}$,

where $p$ is the pressure, $c$ is the velocity, and $h$ is the geodetic height. Here, indices 1 and 2 refer to the suction and the pressure sides, respectively. The diameters of the suction and pressure sides are equal for both pumps, thus the second term of the right hand side is zero. $p_{2}$ is calculated as the average of the measured signal of the transducer, while $p_{1}$ is measured by a U-tube mercury-filled manometers for both pumps.

The efficiency of the pumps is calculated as

$\eta=\frac{P_{u}}{P_{i n}}=\frac{H Q \rho g}{P_{i n}}$,

where $P_{i n}$ is the input power and $P_{u}$ is the useful power. It is important to note that the input powers are measured differently on the two equipment. In the case of pump "A", the mechanical input power can be measured directly by balancing the motor. This means that the driving motor is fixed on its bearings, thus the housing can rotate. The torque acting on the shaft is proportional to the torque acting on the house, which can be measured by balancing it to a steady state. In contrast, in the case of pump " $B$ ", only the measurement of the electric power of the motor was possible due to its compactness. This means that in the case of pump "A", efficiency refers to only the pump itself, but in the case of pump " $B$ " it belongs to the motorpump unit as a whole. This results in a slightly different definition for the efficiencies of the two pumps, but it has no effect to the drawn conclusions in the next sections.

The uncertainties of the estimated quantities were calculated based on the laws of error propagation. The highest absolute errors of the flow rate, head and efficiency were $\Delta Q= \pm 0.51 \mathrm{~L} / \mathrm{s}, \Delta H= \pm 0.9 \mathrm{~m}, \Delta \eta=0.04$, respectively. During the evaluation of the results, we will focus on the amplitude spectra of the measured pressure signals; therefore, detailed uncertainty analysis is omitted and error bars on the diagrams are not indicated.

The strategy to present the results is to calculate the spectrum of the pressure signal on the pressure side along the characteristic curves at several operation points applying different rotational speeds. In this way, one series of spectra can be associated to each characteristic curves. We made measurements on 17 different rotational speeds from 630 to $30001 / \mathrm{min}$ on pump "A", and 8 different rotational speeds from 1300 to $3000 \mathrm{l} / \mathrm{min}$ on pump "B". The flow rates were in the range of 0 to $Q_{\max }$, where $Q_{\max }$ is the flow rate at a totally opened valve with value depending on the current rotational speed. Three measured characteristic curves of the pumps can be seen in Fig. 2. The best efficiency point at a certain rotational speed is indicated by the blue dot. These points define the nominal flow rate $\left(Q_{N}\right)$ at each rotational speed. 

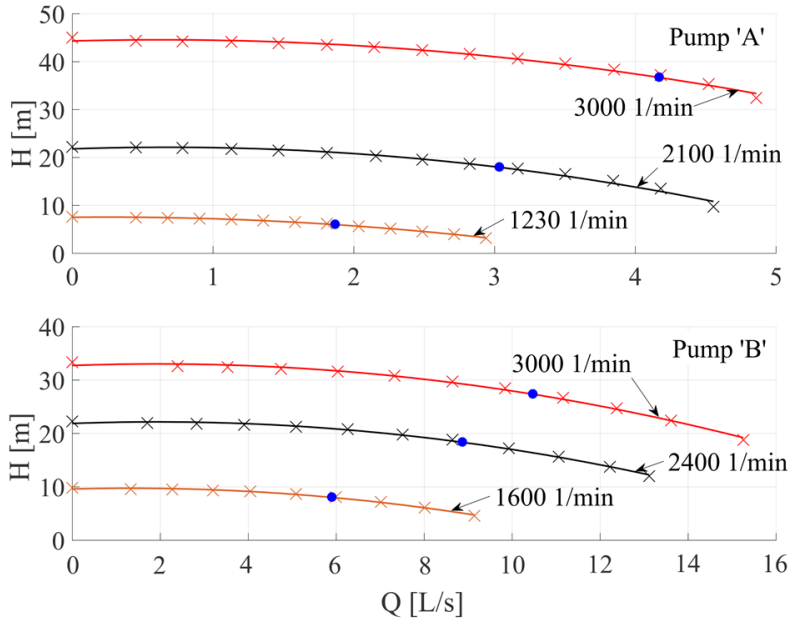

Fig. 2 Three characteristic curves of the pumps with pump " $A$ " on top, and pump " $B$ " on bottom. The best efficiency points are indicated by the blue dots on each rotational speed. These points determine the nominal flow rates. Pressure signals were taken at every operation point marked by the crosses.

\section{Analysis process}

The basic elements of the analysis are the spectra obtained by Fourier transforming the measured pressure signals. An example is shown in Fig. 3 for pump " $A$ " at rotational speed $n=2100 \mathrm{l} / \mathrm{min}$ and flow rate $Q=2.49 \mathrm{~L} / \mathrm{s}$. The sharp peaks and their harmonics can provide valuable information for condition monitoring of the machines discussed in more details during the next sections.
The measured spectra at the operation points along a characteristic curve can be organized into a three dimensional waterfall diagram, where the 2D spectra are plotted as function of the flow rate. In the following, it is helpful to make use of dimensionless forms of the frequency and the flow rate [7] indicated by an asterisk. Therefore, we let $Q^{*}=\frac{Q}{Q_{N}}$,

and

$$
f^{*}=\frac{f}{f_{r}},
$$

where $f_{r}$ is the current rotational speed in $\mathrm{Hz}$ unit. With this nondimensionalization, $Q^{*}=1$ belongs to the best efficiency point. Similarly, $f^{*}=1$ refers to the rotational speed.

Fig. 4 shows a typical waterfall diagram of pump " $A$ " at $n=30001 / \mathrm{min}$, in which the evolution of the peak amplitudes as a function of the flow rate can be clearly seen. The series of such diagrams with varying rotational speed gives a good overall insight into the behavior of the equipment in a wide range of the operation parameters. The waterfall diagrams that are corresponding to all the measured rotational speeds are shown in the Appendix.

Before a detailed analysis, it is important to summarize that what kind of basic physical effects can be associated to certain features of the spectra and the waterfall diagrams. These are divided mainly into two groups: mechanical and hydrodynamical [8], see also Table 1. Mechanical effects can be productional or constructional failures like
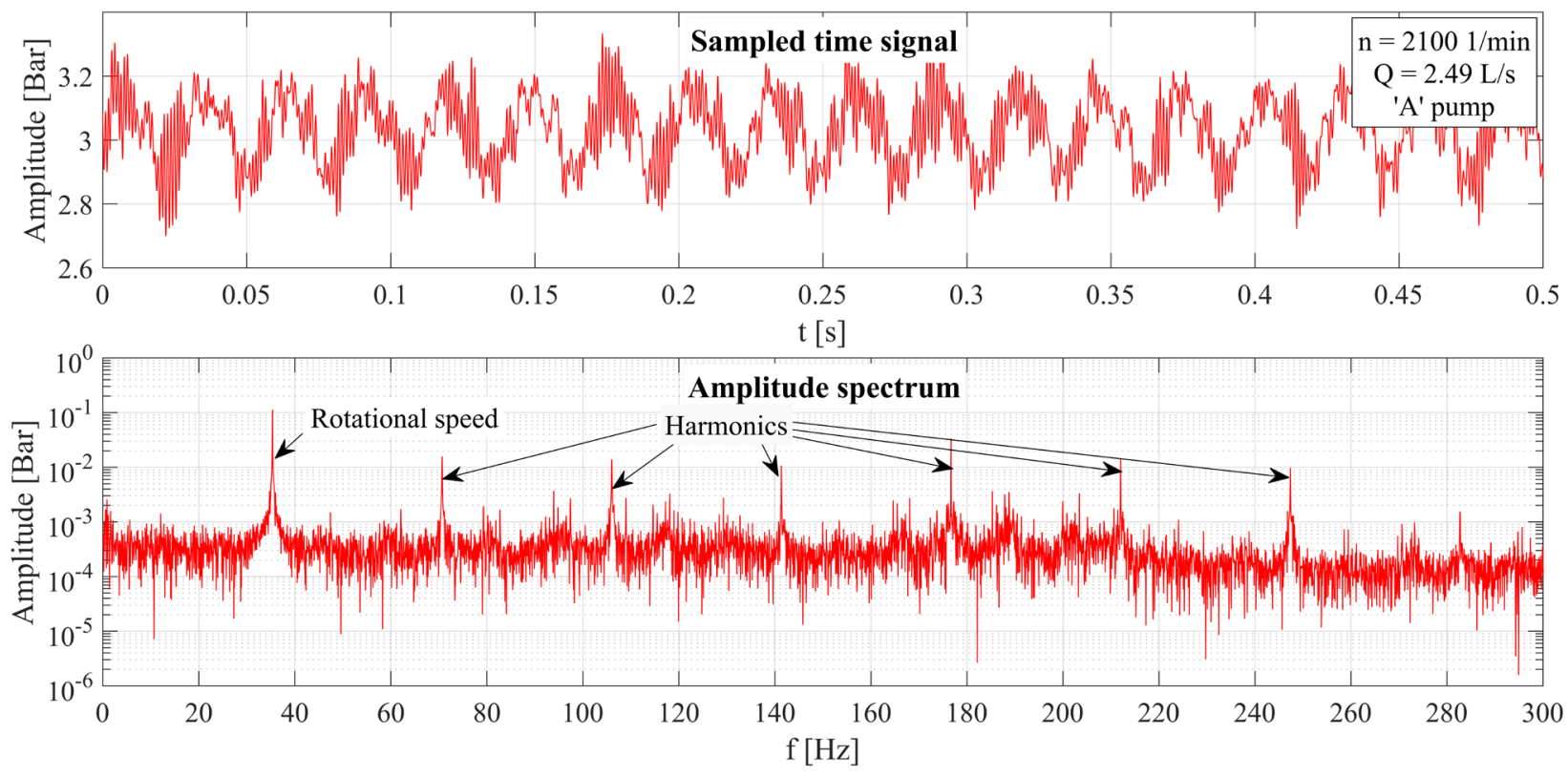

Fig. 3 Time signal and amplitude spectrum in an operating point of pump " $A$ " ( $n=21001 / \mathrm{min}, Q=2.49$ L/s). The sharp peaks and wideband regions contain useful information about the condition of the pump. 


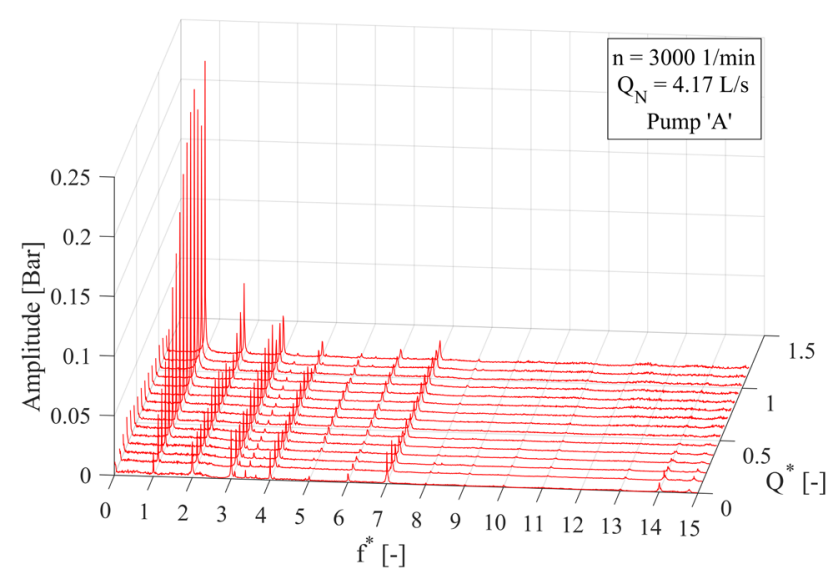

Fig. 4 Waterfall diagram of pump " $A$ " at $n=30001 / \mathrm{min}$. A waterfall diagram contains overall information about the behaviour of the pump on a specific rotational speed.

Table 1 Summary of the physical effects acting on pumps (based on [8])

\begin{tabular}{|c|c|c|}
\hline & Physical effect & Frequency component \\
\hline \multirow{4}{*}{ 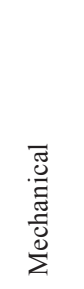 } & unbalance & $f_{r}$ \\
\hline & bent shaft & $(1-2) f_{r}$ \\
\hline & pump and motor misalignment & $(1-4) f_{r}$ \\
\hline & bearing misalignment & $(2-3) f_{r}$ \\
\hline \multirow{4}{*}{ 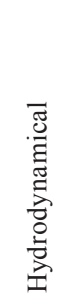 } & internal assembly looseness & $(2-6) f_{r}$ \\
\hline & impeller-volute interaction & $f_{B P F}$ \\
\hline & non-uniform flow distribution & $f_{B P F}$ \\
\hline & cavitation & $\begin{array}{l}\text { high frequency } \\
\text { wideband region }\end{array}$ \\
\hline \multirow{2}{*}{ Other } & motor instability & frequency modulation \\
\hline & non-linearity & upper harmonics \\
\hline
\end{tabular}

unbalance, bent shaft, pump and motor misalignment or bearing failures. These types of effects have a common nature that they cause sharp peaks in the spectra at $f_{r}$ and its integer multiples (up to $2 \times-6 \times$ ). If the presence of any mechanical effects is significant, these peaks dominate the spectra. Hydrodynamical effects are mainly the impact of impeller-volute interaction, cavitation or non-uniform flow distribution around the blades. They are presented even in a well-designed pump. Mostly, these effects cause wideband regions in the spectrum, or a sharp peak at the so-called blade passage frequency (BPF) [8], defined as
$f_{B P F}=z \cdot f_{r}$,

where $z$ is number of the blades. This frequency is generated mainly due to the gap between the impeller and the tongue of the volute. The optimal size of the gap is around 4-6\% of the impeller diameter. Decreasing the gap results in higher efficiency, but the pressure fluctuations and the transmitted noise also increases $[10,11]$.

It has to be mentioned that due to instability reasons, the constant rotational speed of the driving motor is not always guaranteed during a measurement causing a phenomenon called frequency modulation [12]. As a result, small amplitudes appear in the spectrum slightly under and above of the frequency $f_{r}$ and its multiples.

Another important phenomenon have to be considered is the nonlinearity of the system, which causes peaks also at integer multiples of $f_{r}$ called harmonics. It is quite difficult to separate whether a frequency component is generated directly by a physical effect or it is only a harmonic of some lower frequency. Fortunately, the harmonics of a physical effect usually have exponentially decreasing amplitudes [13]. Therefore, the non-monotonic decrease of the peaks at integer multiples of $f_{r}$ indicates the presence of mechanical vibrations in addition to unbalance.

It is important to mention that the values of Table 1 correspond to vibration measurements on the housings of the bearings. However, every mechanical vibration propagates into the fluid as pressure waves, consequently the pressure values (and all other fluid quantities, too) fluctuate with the same frequencies as the mechanical vibration itself. This wave propagation mechanism is mostly nonlinear and the waveform is deformed during the propagation, but this phenomenon can be considered as a part of the aforementioned nonlinear effects. In this sense, it can be declared that the spectra of pressure signals are mostly resemble to those of vibration measurements.

Naturally, the final form of a spectrum is resulted by the sum of every operating effect. If a frequency component is generated by multiple different physical sources, their amplitudes are added. In general, it is hard to separate these individual effects; however, it can be stated that if a pump contains many mechanical failures, the waterfall diagrams are dominated by sharp peaks at $f_{r}$ and its integer multiples. Otherwise, mostly broadband regions and a peak at the blade passage frequency are can be observed in the waterfall diagrams as a result of hydrodynamical effects. 


\section{Results}

First of all, it is important to emphasize that most of the statements in this paper are often only assumptions that need more detailed investigation, but they can be a good basis of further research and applications.

\subsection{Pump " $A$ "}

As it is mentioned before, pump " $A$ " is a much more used, worn pump. Consequently, the possibility of many mechanical failures and misalignments are very likely. Fig. 5 shows the waterfall diagram of pump " $A$ " at low rotational speed $n=630 \mathrm{l} / \mathrm{min}$. It can be observed that a sharp peak dominates the spectra at $f^{*}=1$, most probably due to unbalance. The amplitude of this peak increases monotonically with the flow rate. The harmonics are presumably the effect of nonlinearity due to their exponentially and monotonically decreasing amplitudes. The significant peak at $f^{*}=7$ is the blade passage frequency as pump " $A$ " has seven blades. According to Fig. 5, pump " $A$ " might be heavily loaded with unbalance. Similar spectra can also be observed also at $n=760 \mathrm{l} / \mathrm{min}$ (see the Appendix for the waterfall diagram).

An additional interesting phenomenon appears on the waterfall diagram at $n=920 \mathrm{l} / \mathrm{min}$ shown in Fig. 6 . High mechanical and acoustic noise was experienced during the measurement. The mechanical vibrations were so heavy that it was audible and caused tremors in the laboratory. This vibration can be easily noticed in the waterfall diagram, as a low-amplitude wideband zone is suppressing the peak at the blade passage frequency (at $f^{*}=7$ ). This is probably the result of the experienced heavy vibrations on the machine. Observe, however, that the other peaks at $f^{*}=4$ or 5 can still be seen, perhaps as a result of the increasing effect of some other mechanical effects. It can be also noticed that the peaks at $f^{*}=2$ and 3 are much weaker resulting in a nonmonotomic nature of the harmonics. That is, the harmonics are influenced by additional (external) sources besides nonlinearity. Interestingly the heavy vibrations disappear at high flow rates. Above $Q^{*}=1.5$, the spectra is quite similar to the one presented at $n=630 \mathrm{l} / \mathrm{min}$ (see again Fig. 5); that is, the harmonics of $f^{*}=1$ are decreasing exponentially due to the nonlinearity. The flow rate dependence of such a heavy mechanical vibration can imply mounting problems of the impeller. As the flow rate varies, the unbalanced axial and radial forces acting on the impeller changes as well. In case of bad fitting, these forces might "kick" the system to a heavy vibrational state. The traces of this serious mechanical effect is presented in the waterfall diagrams approximately between rotational speeds $n=720 \mathrm{l} / \mathrm{min}$ and $n=1650 \mathrm{l} / \mathrm{min}$.

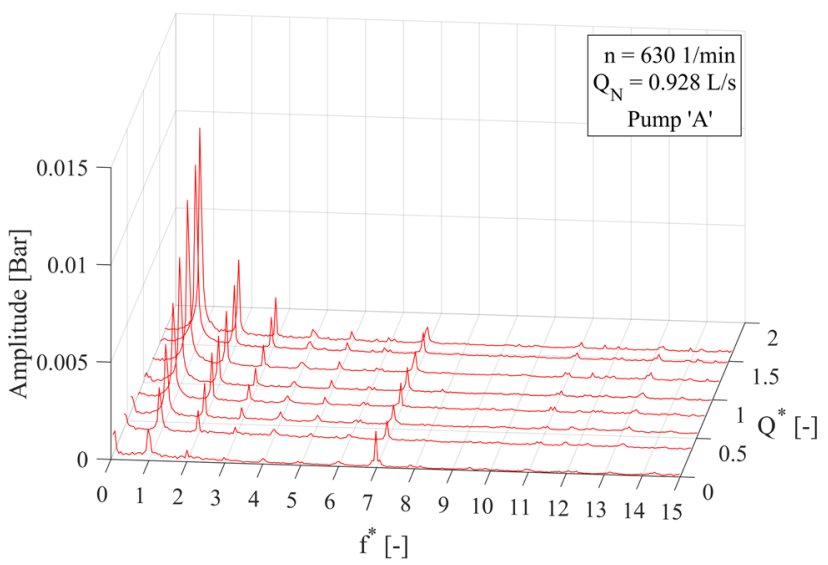

Fig. 5 Waterfall diagram of pump " $A$ " at $n=6301 / \mathrm{min}$. A dominant excitation (most probably unbalance) can be observed at $f^{*}=1$. Upper harmonics are decreasing exponentially. A slightly dominant peak can be seen at $f_{B P F}$.

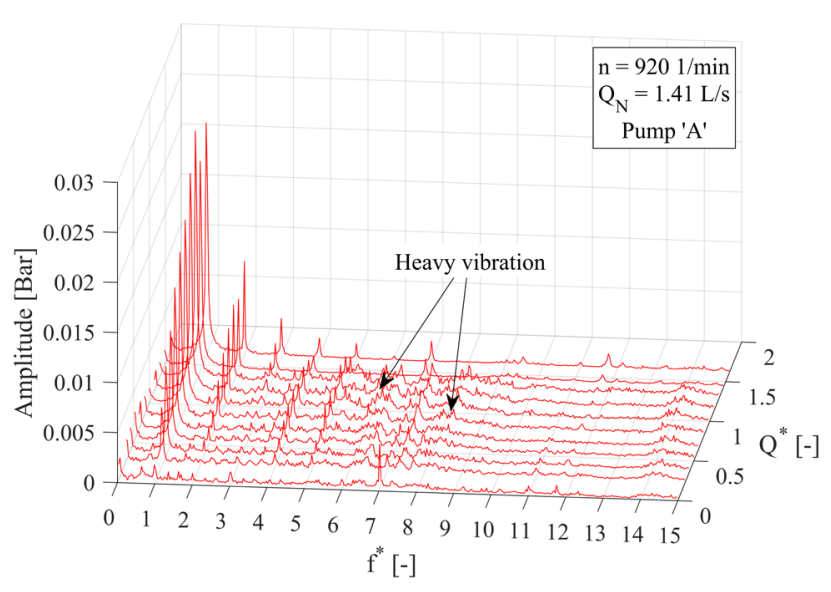

Fig. 6 Waterfall diagram of pump " $A$ " at $n=9201 / \mathrm{min}$. Heavy mechanical and acoustic noise occurred during the measurements, which can be seen in the spectra. This phenomenon disappears as flow rate increases.

On some specific rotational speeds, due to control issues, the driving motor is unable to keep the rotational speed at a constant value, but it fluctuates with low frequency resulting in the aforementioned frequency modulation. This effect can be noticed for instance at $n=1350 \mathrm{l} / \mathrm{min}$ shown in Fig. 7 . It can be clearly seen that near the sharp peaks at multiples of $f^{*}=1$, smaller "spikes" appear in the spectra that is a presumable indicator for frequency modulation [11]. This phenomenon appears over the range of the rotational speed between $n=1350 \mathrm{l} / \mathrm{min}$ and $n=1650 \mathrm{l} / \mathrm{min}$. If stable operation is mandatory, this range should be avoided.

At higher rotational speeds, from approximately $n=1820 \mathrm{l} / \mathrm{min}$ up to $n=3000 \mathrm{l} / \mathrm{min}$, the integer multiples of $f^{*}=1$ in the spectra does not decrease exponentially as can be seen in Fig. 8 at $n=25501 / \mathrm{min}$. This plausibly 


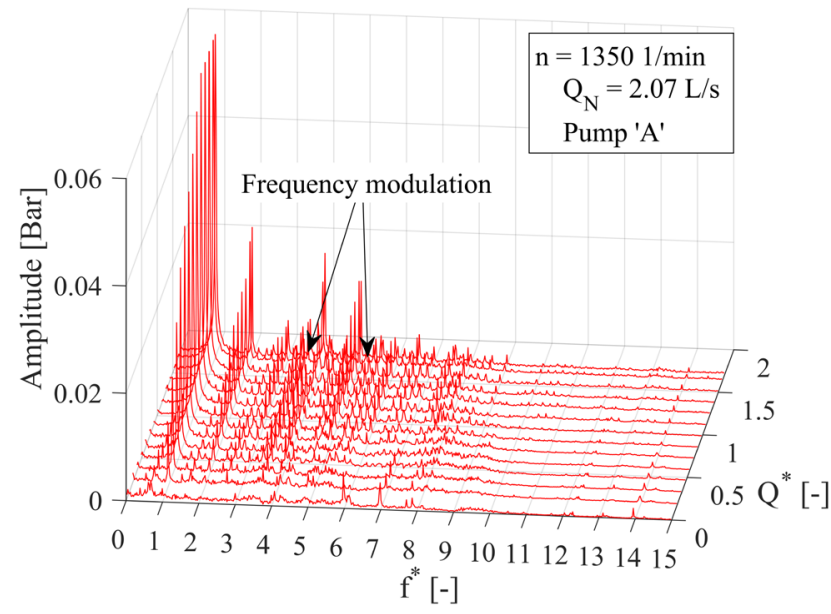

Fig. 7 Waterfall diagram of pump " $A$ " at $n=13501 / \mathrm{min}$. Heavy frequency modulation appears at certain rotational speeds due to control issues.

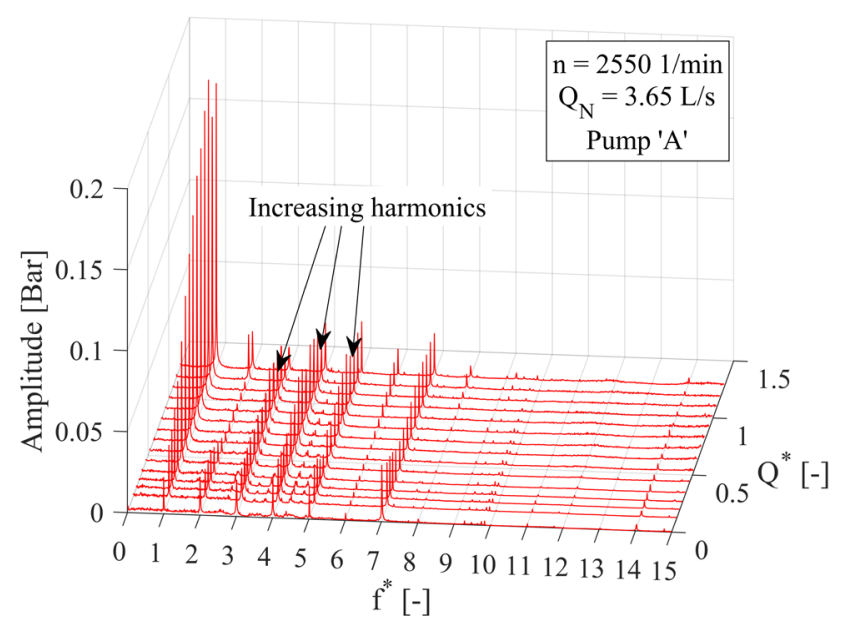

Fig. 8 Waterfall diagram of pump " $A$ " at $n=22501 / \mathrm{min}$. Effects of other mechanical excitations become stronger, as multiples of $f^{*}=1$ does not decrease exponentially.

means that the effect of other mechanical failures (other than the unbalance) that excite on multiples of $f_{r}$ tend to be more and more significant on higher rotational speeds. A potential reason can be that the mechanical forces are proportional to the square of the rotational speed in general; thus, the effect of such mechanical issues become notable only at higher rotational speeds.

Generally, it can be stated that all of the waterfall diagrams of pump "A" are mostly dominated by heavy mechanical effects such as unbalance, bent shaft, assemble looseness, etc. As a result, hydrodynamical noise is buried in the spectra by the sharp peaks caused by the heavy mechanical effects.

\subsection{Pump "B"}

In the case of pump " $B$ ", since it is a less used and newer machine, we expect less mechanical effects on the spectra. Fig. 9 shows a waterfall diagram of pump "B" at low $n=1300 \mathrm{l} / \mathrm{min}$ rotational speed. It can be clearly seen that the spectra is quite different from the ones of pump " $A$ ". There is no noticeable sharp peak at $f^{*}=1$ or its integer multiples, meaning that mechanical effects are conceivably negligible in this case. On the other hand, a wideband region can be observed at low frequencies up to $f^{*} \approx 4$. The only sharp peak appearing in the waterfall diagram is at $f^{*}=6$, which is the blade passage frequency. This might also confirm that hydrodynamical effects are more significant in the case of pump " $B$ ". Observe also that the amplitudes in the waterfall diagram near the same rotational speed for pump " $A$ " are an order of magnitude higher (compare with Fig. 7).

The only rotational speed where sharp peaks appear on pump " $B$ " is at $n=1600 \mathrm{l} / \mathrm{min}$, see in Fig. 10. Here, a peak at $f^{*}=1$ can be noticed, similarly to the general behavior of pump " $A$ ". It is very probable that in this case, the natural (or critical) frequency of the system is excited, which is definitely an undesired operation as it can cause unnecessary load on the bearings as well as other mechanical parts of the pump. An interesting question is whether this situation could have been avoided by proper design of the pump. The amplitudes of the harmonics are decreasing exponentially possibly indicating the effect of the nonlinearity.

At higher rotational speeds, the sharp peak at $f^{*}=1$ disappears. It can be observed at $n=2650$ 1/min on Fig. 11 that the wideband region dominates the spectra again up to $f^{*} \approx 4$. In addition, a sharp peak is presented at

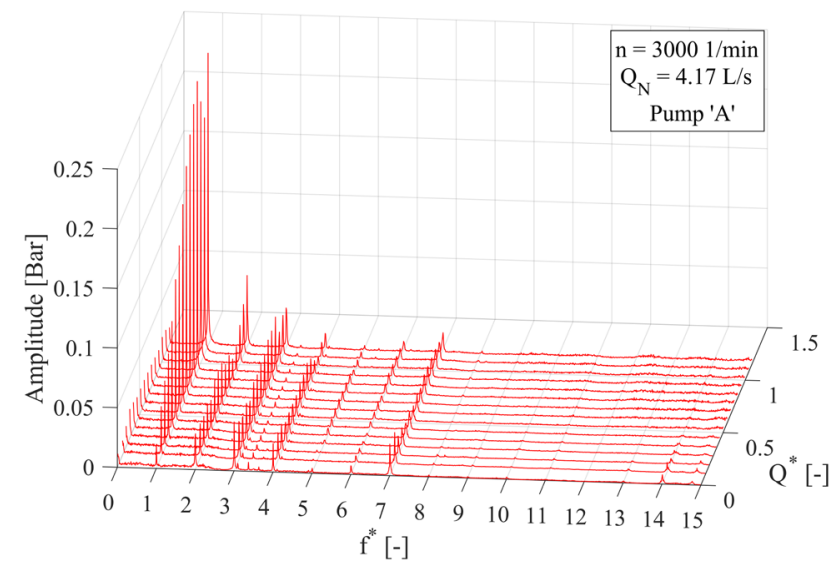

Fig. 9 Waterfall diagram of pump " $B$ " at $n=13001 / \mathrm{min}$. There is no noticeable sharp peak at $f^{*}=1$ or its multiples, but a wideband region can be observed. 


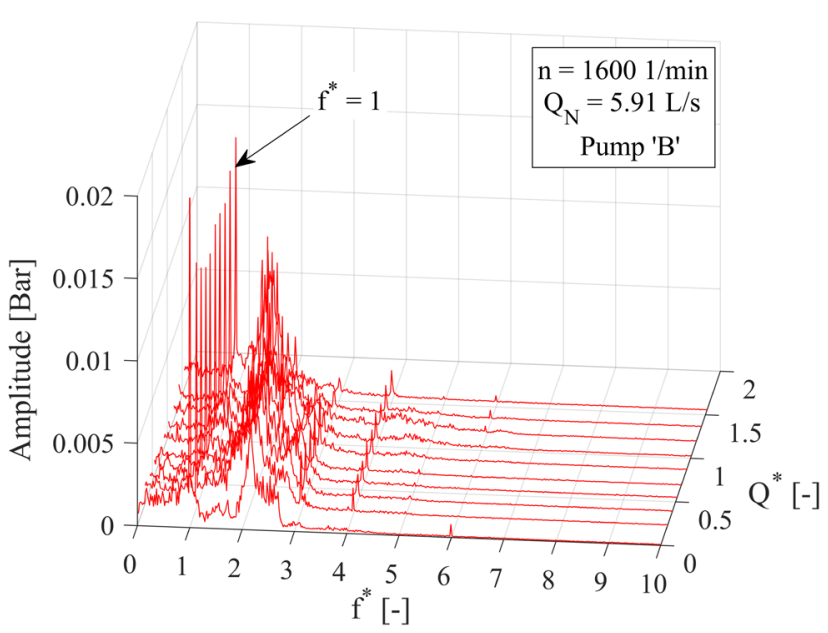

Fig. 10 Waterfall diagram of pump " $B$ " at $n=16001 / \mathrm{min}$. This is the only rotational speed where sharp peaks appear at $f^{*}=1$ and its multiples, due to one of the natural frequencies of the system.

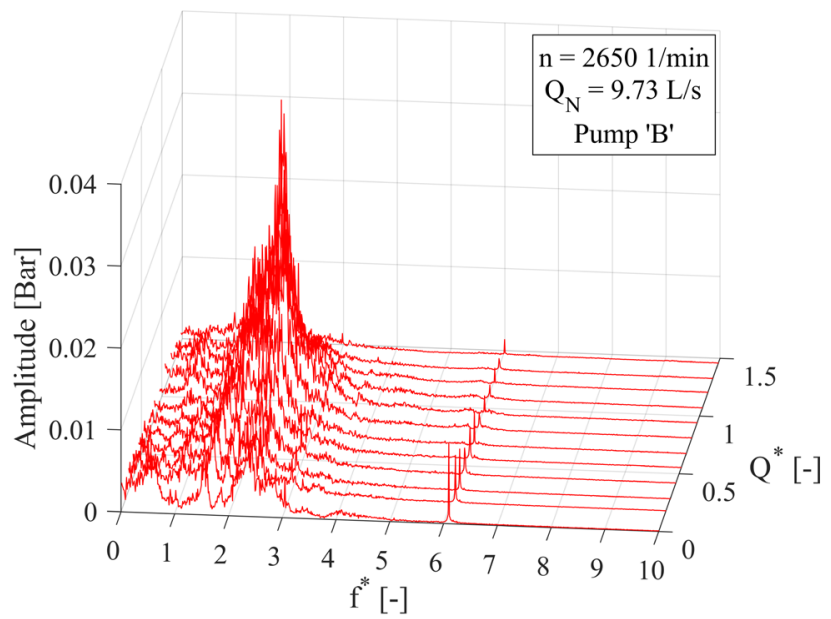

Fig. 11 Waterfall diagram of pump " $B$ " at $n=26501 / \mathrm{min}$. A wideband region dominates the spectra up to $f^{*} \approx 4$. The only sharp peak is the one at the blade passage frequency.

$f^{*}=6$ (blade passage frequency). The amplitude of this frequency tends to decrease towards $Q^{*}=1$, which is in good agreement with results of other researchers [7, 14].

In summary, it can be concluded that the spectra in pump " $B$ " is most likely dominated by hydrodynamical effects, since there are no sharp peaks in the spectra at $f^{*}=1$ and its integer multiples in general (except at $n=1600 \mathrm{l} / \mathrm{min}$ ). In contrast, the waterfall diagrams contain wideband regions, and only one peak at the blade passage frequency, which may also confirm the dominance of hydrodynamical effects. The detailed analysis of this hydrodynamical wideband frequency region is beyond the scope of the present study as it is focusing on the vibration monitoring and health issues of pumps, which mainly have mechanical origin.

\section{Conclusions}

By measuring the pressure signals on two different centrifugal pumps, conclusions were drawn about their health condition through dimensionless waterfall diagrams along their characteristic curves. As a result, it has been found that pump " $A$ ", which is a more used, older machine, is most probably loaded by several mechanical vibrations, since all of the spectra are dominated by sharp peaks at $f_{r}$ and its integer multiples. It can be clearly seen that a quite significant load is acting on the pump corresponding to $f^{*}=1$, which is seemingly due to the unbalance of the impeller. On lower rotational speeds, the harmonics of $f^{*}=1$ are decreasing exponentially supposedly because of the effect of non-linearity (see again the discussion of Section 3). However, at higher rotational speeds, these multiples gradually become more significant; that is, other physical effects (not only the unbalance) that generate peaks between $f^{*}=2-4$ may have more influence at higher rotational speeds. The instability of the operation of the driving motor was also identified as a frequency modulated spectra.

In the case of pump " $B$ ", which is a much newer machine, it has been found that with the exception of one rotational speed $(n=1600 \mathrm{l} / \mathrm{min})$, mostly hydrodynamical effects impact on the spectra, due to the precise assembly and installation of the system. Most of the waterfall diagrams are dominated by a wideband region, and a sharp peak only at the blade passage frequency.

Comparing the waterfall diagrams of the two pumps, one can observe that pressure fluctuations on pump " $A$ " are significantly higher. For example, comparing Figs. 7 and 9, the maximum value of the amplitudes are approximately 0.2 and 0.006 bar for pump " $A$ " and " $B$ ", respectively, which is more than one order of magnitude difference. Consequently, it might become possible to separate mechanical and hydrodynamical effects acting on a centrifugal pump. It is clear that mechanical effects cause sharp peaks in the spectra at $f_{r}$ and its integer multiples up to $f^{*}=2-4$. Moreover, if the harmonics decrease exponentially then those multiples are only the effects of non-linearity.

In summary, we have the opportunity to identify the appearance of a mechanical failure on a pump. By continuously monitoring the amplitudes of the peaks at $f_{r}$ or its integer multiples, and compare them to former ones, it is possible to act in time and perform maintenance on the system to prevent heavy structural damage. The authors are aware of the fact that in order to apply this method confidently, it has to be improved further, for example comparing with vibration measurements, or generating some of the mentioned physical effects intentionally. 


\section{References}

[1] Gülich, J. F. "Centrifugal Pumps", 3rd ed., Springer, Berlin Heidelberg, Germany, 2014.

https://doi.org/10.1007/978-3-642-40114-5

[2] Behzad, M., Bastami, A. R., Maassoumian, M. "Fault Diagnosis of a Centrifugal Pump by Vibration Analysis", In: $7^{\text {th }}$ Biennial Conference on Engineering Systems Design and Analysis, Manchester, England, 2004, pp. 221-226. https://doi.org/10.1115/ESDA2004-58534

[3] Albraik, A., Althobiani, F., Gu, F., Ball, A. "Diagnosis of Centrifugal Pump Faults Using Vibration Methods", Journal of Physics: Conference Series, 364, article ID: 012139, 2012. https://doi.org/10.1088/1742-6596/364/1/012139

[4] Jensen, J., Dayton, K. "Detecting Cavitation in Centrifugal Pumps", Orbit, 21(2), pp. 26-30, 2000.

[5] Čudina, M. "Detection of Cavitation Phenomenon in a Centrifugal Pump Using Audible Sound", Mechanical Systems and Signal Processing, 17(6), pp. 1335-1347, 2003. https://doi.org/10.1006/mssp.2002.1514

[6] Hungarian Standards Institution "MSZ EN ISO 5167:2003. Measurement of fluid flow by means of pressure differential devices inserted in circular cross-section conduits running full. Part 1: General principles and requirements (ISO 5167-1:2003)", Budapest, 2003.

[7] Parrondo-Gayo, J. L., González-Pérez, J., Fernández-Francos, J. "The Effect of the Operating Point on the Pressure Fluctuations at the Blade Passage Frequency in the Volute of a Centrifugal Pump", Journal of Fluids Engineering, 124(3), 2002, pp. 784-790. https://doi.org/10.1115/1.1493814

[8] Scheffer, C. "Session Five: Pump Condition Monitoring Through Vibration Analysis", In: PUMPS: Maintenance, Design, and Reliability Conference 2008 - IDC Technologies, Johannesburg, Republic of South Africa, 2008, pp. 1-20.
[9] Zhang, N., Yang, M., Gao, B., Li, Z., Ni, D. "Experimental and Numerical Analysis of Unsteady Pressure Pulsation in a Centrifugal Pump with Slope Volute", Journal of Mechanical Science and Technology, 29(10), 2015, pp. 4231-4238. https://doi.org/10.1007/s12206-015-0919-y

[10] González, J., Parrondo, J., Santolaria, C., Blanco, E. "Steady and Unsteady Radial Forces for a Centrifugal Pump With Impeller to Tongue Gap Variation", Journal of Fluids Engineering, 128(3), 2005, pp. 454-462. https://doi.org/10.1115/1.2173294

[11] Solis, M., Bakir, F., Khelladi, S. "Pressure Fluctuations Reduction in Centrifugal Pumps: Influence of Impeller Geometry and Radial Gap", In: Fluids Engineering Division Summer Meeting, FEDSM2009, Vail, Colorado, USA, 2009, pp. 253-265. https://doi.org/10.1115/FEDSM2009-78240

[12] Guicking, D. "Schwingungen: Theorie und Anwendungen in Mechanik, Akustik, Elektrik und Optik", (Vibration: Theory and applications in mechanics, acoustics, electrics and optics), 1st ed., Springer Vieweg, Wiesbaden, Germany, 2016. (in German) https://doi.org/10.1007/978-3-658-14136-3

[13] Strogatz, S. H. "Nonlinear Dynamics and Chaos: With Applications to Physics, Biology, Chemistry, and Engineering", 1st ed., Perseus Books Group, Reading, Massachusetts, 1994.

[14] Hodkiewicz, M. R., Norton, M. P. "The Effect of Change in Flow Rate on the Vibration of Double-Suction Centrifugal Pumps", Proceedings of the Institution of Mechanical Engineers, Part E: Journal of Process Mechanical Engineering, 216(1), 2002, pp. 47-58. https://doi.org/10.1243/095440802760075030 


\section{Appendix}

Here is the calibration diagram of the pressure transducer with its confidence bands corresponding to $95 \%$ significance level, and the equation of calibration.

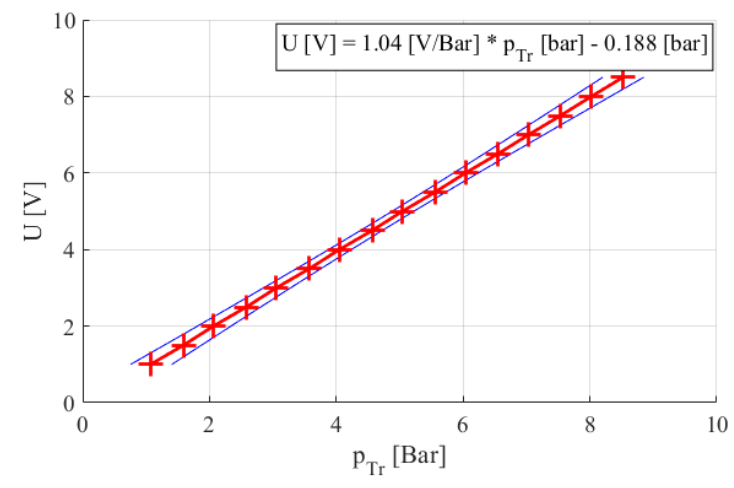

Fig. A1 Calibration diagram of the applied pressure transducer with its confidence bounds corresponding to $95 \%$ confidence level.

Next, we present all measured waterfall diagrams for the two pumps:

Pump "A":
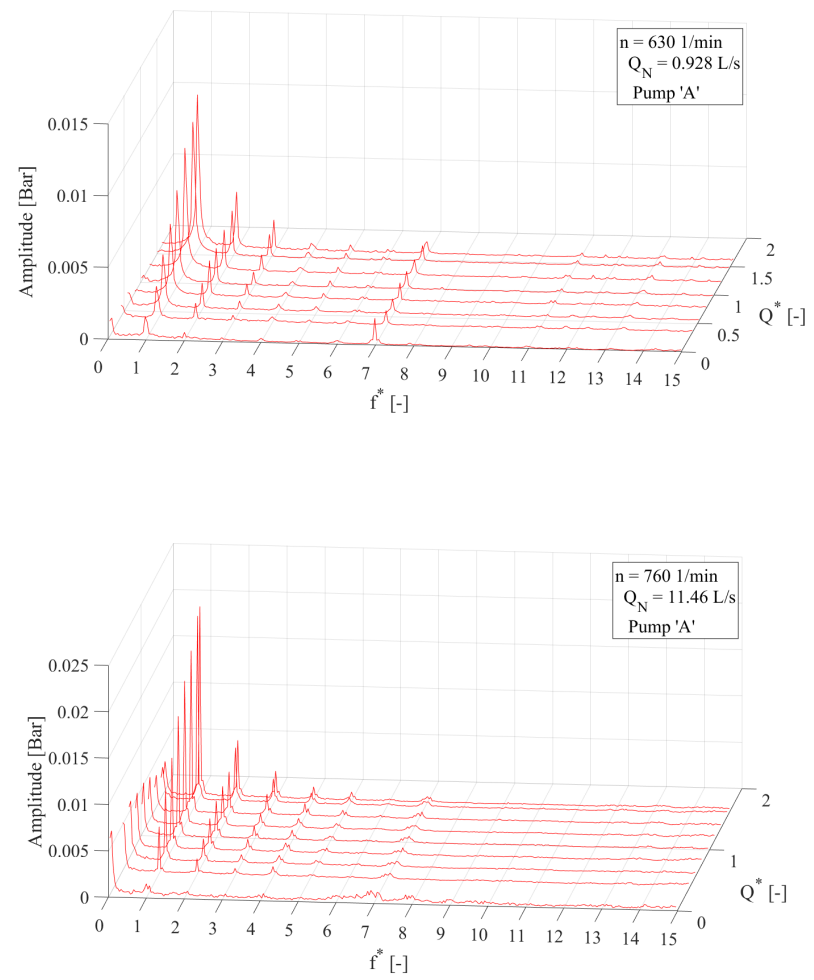


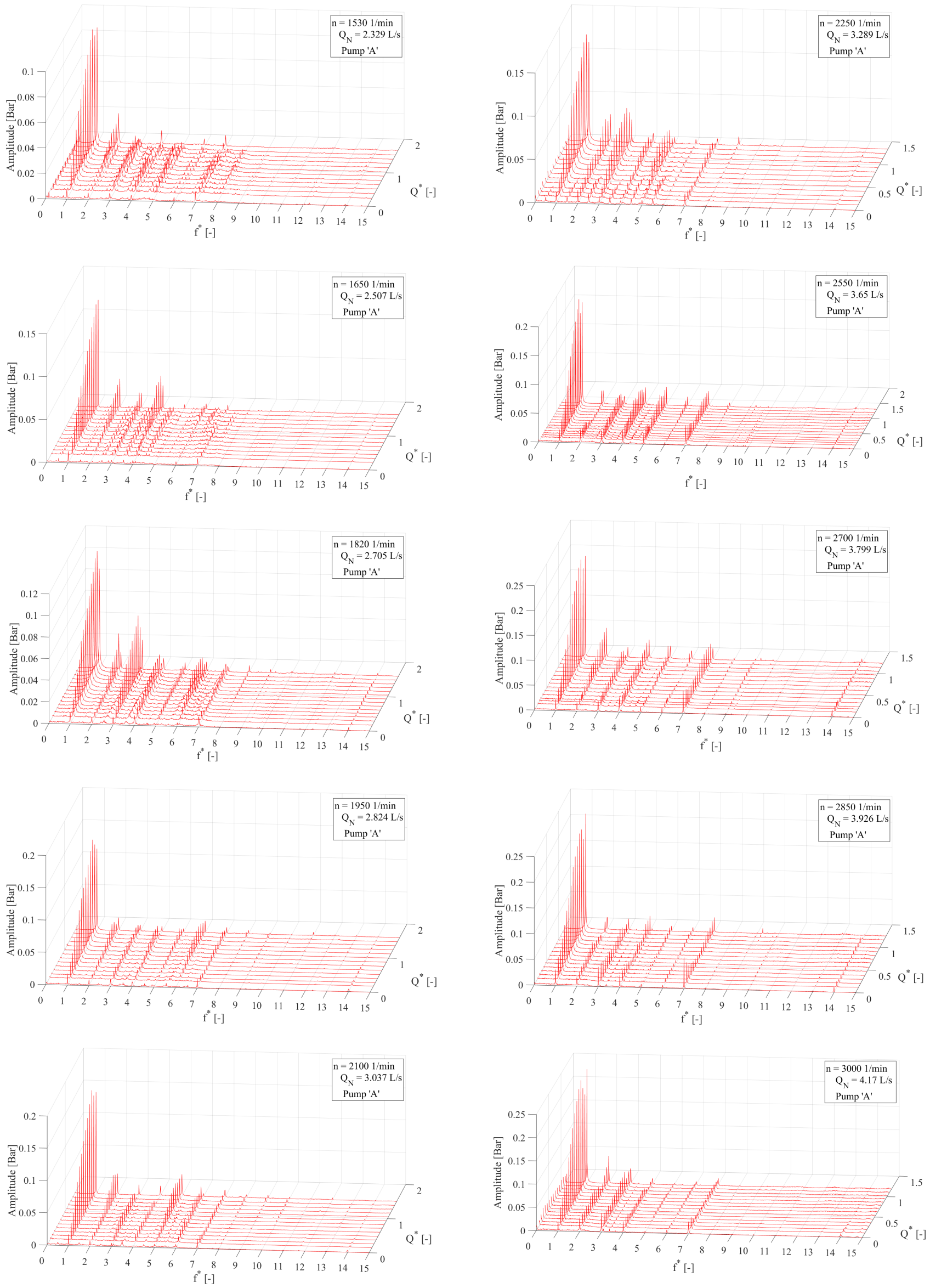


\section{Pump "B":}
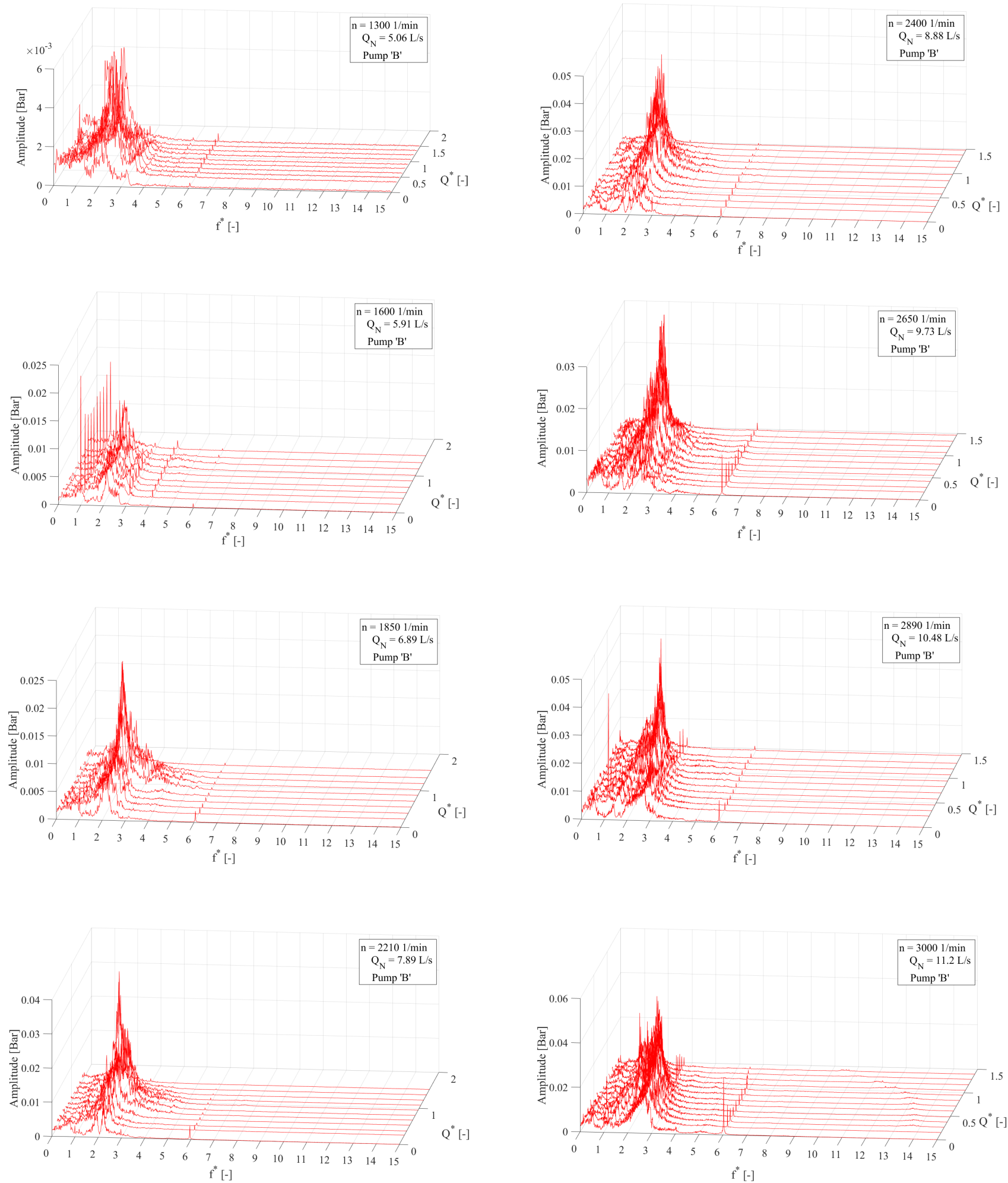